\title{
Experimental Determination of the Equilibrium Conditions of Binary Gas Hydrates of Cyclopentane + Oxygen, Cyclopentane + Nitrogen, and Cyclopentane + Hydrogen
}

\author{
Du Jianwei, Liang Deqing,* Li Dongliang, and Li Xinjun \\ Key Laboratory of Renewable Energy and Gas Hydrate, Guangzhou Institute of Energy Conversion, \\ CAS, Guangzhou 510640, China
}

In this work, four-phase hydrate equilibrium data for each of the systems cyclopentane $(\mathrm{CP})+$ water + hydrogen, $\mathrm{CP}+$ water + nitrogen, and $\mathrm{CP}+$ water + oxygen were measured and are reported in the temperature range of 281.3-303.1 $\mathrm{K}$ and the pressure range of 2.27-30.40 MPa. Measurements were made using an isochoric method. Experimental data on the equilibrium conditions of the two systems nitrogen + water and hydrogen + water with $\mathrm{CP}$ are reported over a previously uninvestigated extended temperature span. The hydrate dissociation data for the $\mathrm{CP}+$ water + hydrogen system are compared with some selected experimental data from the literature, and the acceptable agreement demonstrates the reliability of the experimental method used in this work. Finally, the first quadruple phase equilibrium data in the ternary system $\mathrm{CP}+$ water + oxygen have been achieved.

\section{Introduction}

Clathrate hydrates are crystalline inclusion compounds composed of a hydrogen-bonded water host lattice and one or more types of guest molecules. ${ }^{1}$ Hydrates encapsulating two types of guest molecules are specifically called binary hydrates or double hydrates. The occupation of hydrate cages by multiple guest molecules helps maintain the thermodynamic stability of the clathrate hydrate crystalline structure at lower pressures and higher temperatures; for example, the pressure at a specific temperature at which the pure hydrogen hydrate is stable can be decreased by 2 or more orders of magnitude in the presence of tetrahydrofuran (THF)., ${ }^{2,3}$ Lowering the gas hydrate formation pressure at atmospheric temperature is a key issue for the industrial applications involving the storage of gas (e.g., methane, hydrogen, or natural gas) or the separation of mixed gases using hydrates. Therefore, adding a compound to a binary gas + water system as a guest molecule to help stabilize the hydrate cages is a relatively valid method of reducing the synthesis pressures of single gas hydrates. Among the three common hydrate structures, the ratio of small to large cages is greatest in $\mathrm{sH}$ hydrogen hydrates, which means that these materials theoretically have the highest hydrogen storage capacity. However, the formation pressures of $\mathrm{sH}$ binary hydrogen hydrates such as $\mathrm{MCH}$ (methylcyclohexane) + hydrogen hydrate, MTBE (methyl tert-butyl ether) + hydrogen hydrate, 1,1-DMCH (1,1-dimethylcyclohexane) + hydrogen hydrate, ${ }^{4}$ and methylcyclopentane + hydrogen hydrate, ${ }^{5}$ are much higher than those of structure II (sII) binary hydrogen hydrates. sII binary hydrogen hydrates are thus naturally better suited to fulfill the task of storing hydrogen.

Cyclopentane (CP) is known to form a single sII hydrate with water at a temperature near $280 \mathrm{~K}$ at atmospheric pressure. ${ }^{6,7}$ Research on $\mathrm{CP}$ as a guest molecule has been reported for the ternary systems of $\mathrm{CP}+$ water + carbon dioxide, ${ }^{8} \mathrm{CP}+$ water + difluoromethane, ${ }^{9} \mathrm{CP}+$ water + krypton, ${ }^{10} \mathrm{CP}+$ water + methylfluoride, ${ }^{11} \mathrm{CP}+$ water + methane, ${ }^{12,13} \mathrm{CP}+$ water + nitrogen, ${ }^{13} \mathrm{CP}+$ water + hydrogen, ${ }^{14-16}$ and $\mathrm{CP}+$ water +

* To whom correspondence should be addressed. E-mail: Liangdq @ ms.giec.ac.cn. Tel.: +(86)20-87057669. Fax: +(86)20-87057669. hydrogen sulfide. ${ }^{17}$ These experimental results are critical for gas separation using hydrate technology. However, neither research on the $\mathrm{CP}+$ water + oxygen system nor thermodynamic equilibrium data for the binary clathrate hydrate of $\mathrm{CP}$ + water + oxygen is available in the literature.

In the present study, phase equilibrium measurements were implemented to obtain the four-phase hydrate equilibrium data of the $\mathrm{CP}+$ water + hydrogen, $\mathrm{CP}+$ water + nitrogen, and $\mathrm{CP}+$ water + oxygen systems in the temperature range of 281.3-303.1 $\mathrm{K}$ and the pressure range of 2.27-30.40 MPa. The experimental data for the $\mathrm{CP}+$ water + hydrogen system are compared with some selected values from the literature, ${ }^{14-16}$ and the acceptable agreement confirms the accuracy of the experimental apparatus and the feasibility of the isochoric method used in this work. Meanwhile, the experimental data on the equilibrium conditions of double nitrogen and $\mathrm{CP}$ hydrates are reported over a previously uninvestigated extended temperature span, and the first phase equilibrium data for the ternary system of CP + water + oxygen have been achieved. Finally, we discuss in detail the fact that $\mathrm{CP}$ as a potential largemolecule guest substance for structure II hydrates can markedly reduce the binary clathrate hydrate formation pressure with hydrogen, nitrogen, and oxygen as the small-molecule guest substances by changing the thermodynamic conditions.

\section{Experimental Section}

2.1. Preparation of Materials. The materials used in the present study are listed in Table 1, along with their purities, suppliers, and phase states. Hydrogen, oxygen, nitrogen, and $\mathrm{CP}$ were used without any further purification. Deionized water was used after careful degassing. In this work, the fluids used

Table 1. List of Materials Used in the Experiments

\begin{tabular}{lcll}
\hline \multicolumn{1}{c}{ component } & purity $(\%)$ & \multicolumn{1}{c}{ supplier } & phase \\
\hline hydrogen & 99.999 & Foshan Huate Gas Co. & gas \\
nitrogen & 99.999 & Foshan Huate Gas Co. & gas \\
oxygen & 99.999 & Foshan Huate Gas Co. & gas \\
cyclopentane (CP) & 99.5 & $\begin{array}{c}\text { Chengdu Kelong Chemical } \\
\text { Company }\end{array}$ & liquid \\
water (deionized) & & liquid
\end{tabular}


were mixtures of water and $\mathrm{CP}$. As $\mathrm{CP}$ is a low-boiling-point and indissoluble organic substance, the preparation of the $\mathrm{CP}$ rich liquids was done by the following steps to reduce its volatilization: In a typical procedure, $3.729 \mathrm{~g}$ of $\mathrm{CP}$ and 16.271 $\mathrm{g}$ of water were charged into a $50 \mathrm{~mL}$ flask, giving a $\mathrm{CP}$ mole fraction of 0.0556 , which corresponds to a stoichiometric composition for structure II hydrate. Once the $20 \mathrm{~g}$ of mixed liquid was obtained, a stopper was placed on the volumetric flask. Throughout the process, the mass of the system was monitored with a mass balance.

2.2. Experimental Apparatus. The apparatus employed in the present study has been described by Sun et al. ${ }^{12}$ and Liang et al. ${ }^{18}$ The main part of the apparatus is a "full-view" sapphire variable-volume cell with a movable piston, which allows measurement of phase equilibrium curves within the pressure range from 0.1 to $40 \mathrm{MPa}$ with an uncertainty of $\pm 0.024 \mathrm{MPa}$ and within the temperature range from 253 to $399 \mathrm{~K}$ with an uncertainty of $\pm 0.1 \mathrm{~K}$. The test contents were mixed with a stirrer that was driven by a dc motor located at the end of the piston and a magnetic coupling mounted outside the cell. The cell volume was adjusted with the movable piston. The data from the acquisition system were saved at preset sampling intervals on a computer. A detailed schematic figure of the apparatus is presented in ref 18 .

2.3. Experimental Methods and Procedures. For the ternary systems $\mathrm{CP}+$ water + hydrogen, $\mathrm{CP}+$ water + nitrogen, and $\mathrm{CP}+$ water + oxygen, binary hydrate dissociation $P-T$ conditions were determined by the isochoric pressure-search method. ${ }^{18,19}$ The already-prepared CP-rich liquid was introduced into the vacuum sapphire cell. The cell was pressurized to the desired pressure by supplying hydrogen, oxygen, or nitrogen. Then, the system temperature decreased until the hydrate formed, as confirmed through $P-T$ line changes and visual observation. Once the hydrate had formed, the temperature was increased gradually at about $0.1 \mathrm{~K} / \mathrm{h}$, and $4 \mathrm{~h}$ was required to establish equilibrium adequately at each temperature step. The temperature and pressure were measured continuously, and the equilibrium data were plotted. The point at which the slope of the $P-T$ curve sharply changed was considered as the hydrate dissociation point at which all hydrate crystals have dissociated. This was also confirmed by visual observation. In this way, a pressure-temperature diagram was obtained for each experimental run, from which the hydrate dissociation points were determined. ${ }^{20}$

\section{Results and Discussion}

As shown in Figure 1, the measured dissociation conditions of the ternary system $\mathrm{CP}+$ water + hydrogen were studied in the temperature range of $281.3-303.1 \mathrm{~K}$ and the pressure range of 2.27-30.40 MPa. In this system, the $\mathrm{L}_{\mathrm{w}}$ phase is the liquid water phase, the $\mathrm{L}_{\mathrm{c}}$ phase is the liquid $\mathrm{CP}$ phase, the $\mathrm{H}$ phase is the binary hydrogen + $\mathrm{CP}$ hydrate phase, and the $\mathrm{V}$ phase is the hydrogen gas phase. The $\mathrm{H}+\mathrm{L}_{\mathrm{w}}+\mathrm{L}_{\mathrm{c}}+\mathrm{V}$ equilibrium line was achieved by determining the final dissociation point of the hydrate. Following the point of complete hydrate dissociation, conditions entered the $\mathrm{L}_{\mathrm{w}}+\mathrm{L}_{\mathrm{c}}+\mathrm{V}$ region. The dashed line shows the phase boundary of the sII CP + hydrogen clathrate hydrate. The hydrogen + water and $\mathrm{CP}+$ water phase boundaries are also shown for reference.

Measurements of the binary hydrate dissociation conditions for the $\mathrm{CP}+$ water + hydrogen system were carried out not only in the existing pressure-temperature range reported but also in a higher pressure-temperature region. To check the reliability of the experimental apparatus and the isochoric

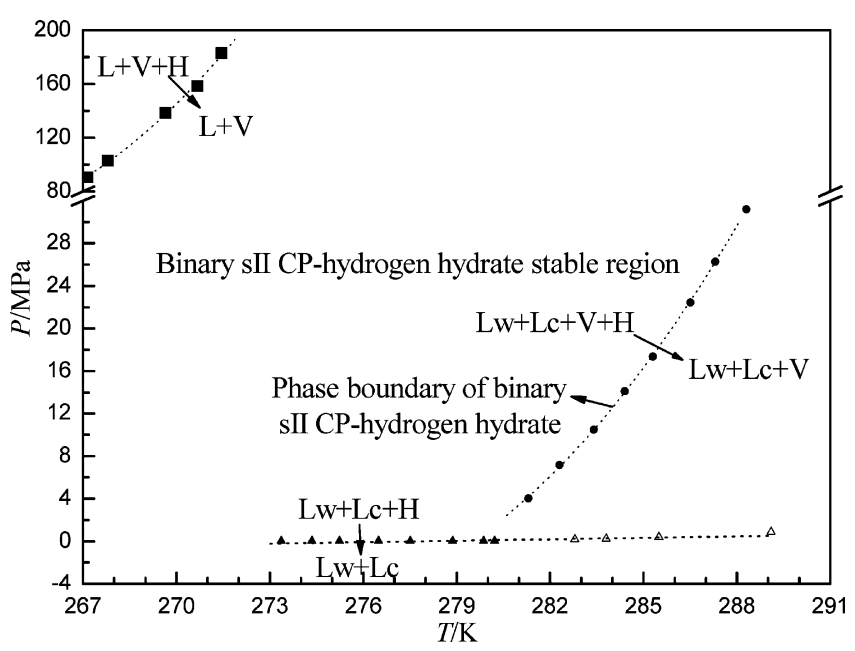

Figure 1. Phase diagram and stability regions: (ם) pure hydrogen hydrate, ref 2; $(\Delta)$ pure CP hydrate, ref $6 ;(\Delta)$ pure $\mathrm{CP}$ hydrate, ref 7; and $(\bullet)$ binary $\mathrm{CP}$-hydrogen hydrate, this work.

Table 2. Binary Hydrate Phase Equilibrium Data for the Systems of CP + Water + Each of the Three Gases (Hydrogen, Nitrogen, and Oxygen) ${ }^{a}$

\begin{tabular}{|c|c|}
\hline$T / \mathrm{K}$ & $P / \mathrm{MPa}$ \\
\hline \multicolumn{2}{|c|}{$\mathrm{CP}+$ Water + Hydrogen } \\
\hline 281.3 & 4.34 \\
\hline 282.3 & 7.30 \\
\hline 283.4 & 10.87 \\
\hline 284.4 & 14.56 \\
\hline 285.3 & 18.29 \\
\hline 286.5 & 23.76 \\
\hline 287.3 & 27.62 \\
\hline 288.3 & 32.53 \\
\hline \multicolumn{2}{|c|}{$\mathrm{CP}+$ Water + Nitrogen } \\
\hline 285.9 & 1.68 \\
\hline 287.5 & 2.49 \\
\hline 290.0 & 3.91 \\
\hline 292.3 & 6.01 \\
\hline 295.2 & 9.39 \\
\hline 297.6 & 13.53 \\
\hline 300.1 & 18.99 \\
\hline 302.0 & 24.45 \\
\hline \multicolumn{2}{|c|}{$\mathrm{CP}+$ Water + Oxygen } \\
\hline 289.4 & 2.27 \\
\hline 290.9 & 3.21 \\
\hline 292.4 & 4.25 \\
\hline 293.7 & 5.36 \\
\hline 294.9 & 6.52 \\
\hline 296.3 & 8.17 \\
\hline 297.9 & 10.24 \\
\hline 299.4 & 12.37 \\
\hline 299.9 & 13.24 \\
\hline 300.4 & 13.91 \\
\hline 301.2 & 15.27 \\
\hline 302.0 & 17.59 \\
\hline 303.3 & 21.69 \\
\hline
\end{tabular}

pressure-search method, four experiments of binary hydrate formation in the $\mathrm{CP}+$ water + hydrogen system were selected from eight phase equilibrium points for comparison with experimental data reported in the literature ${ }^{14-16}$ for the same temperature range. The $P-T$ data obtained in this work are reported in Table 2 and plotted in Figure 2, together with previous data obtained by Zhang and Lee ${ }^{14}$ using high-pressure differential scanning calorimetry and by Cruz Duarte et al. ${ }^{15}$ and Komatsu ${ }^{16}$ employing the same isochoric pressure-search method. Figure 3 shows the deviations between the experimental 


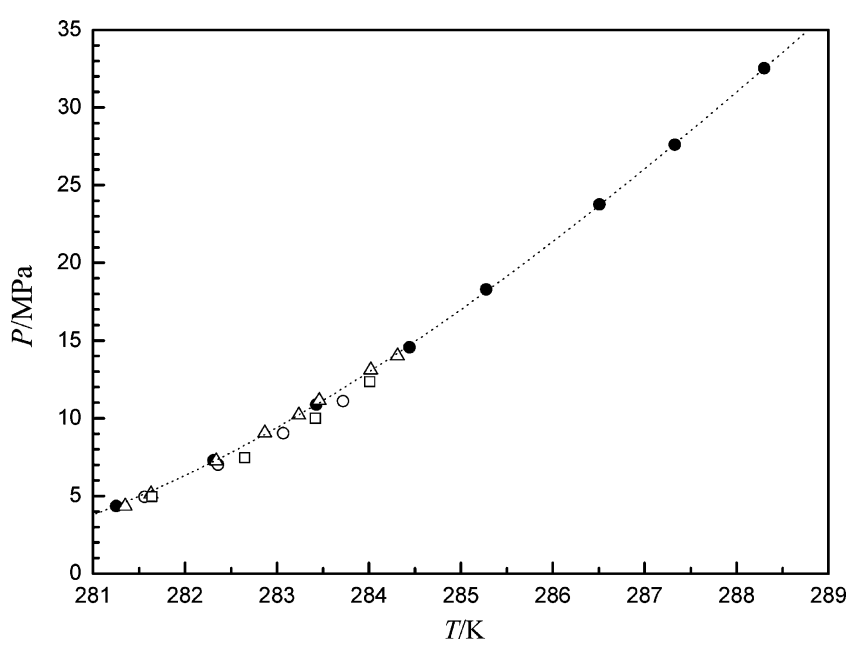

Figure 2. Experimental dissociation conditions for binary $\mathrm{CP}+$ hydrogen clathrate hydrate: $\bullet$, this work; $\bigcirc$, ref $14 ; \square$, ref $15 ; \Delta$, ref 16 .

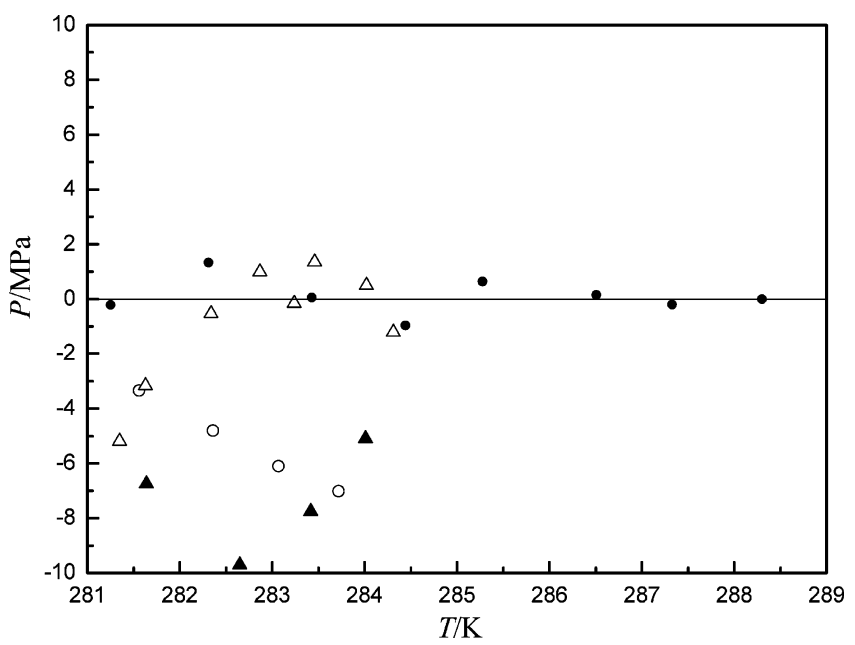

Figure 3. Deviations of the experimental equilibrium pressures for binary hydrate in hydrogen $+\mathrm{CP}+$ water from those calculated by a deviation plot from a three-order polynomial equation in $T / \mathrm{K}$ fit to data $(P /$ MPa) in this work: $P=246618.396791-2551.47 T+8.7822 T^{2}-$ $0.01005509508 T^{3}$. $\bullet$, This work; $\bigcirc$, ref $14 ; \boldsymbol{\Delta}$, ref $15 ; \Delta$, ref 16 .

data reported in the present study and in the literature from the empirical correlation determined by fitting the experimental data obtained in the present study with a three-order polynomial. The deviation is defined as $100 \times\left(p_{\exp }-p_{\text {calc }}\right) / p_{\text {calc }}$, where $p_{\exp }$ is the experimental equilibrium pressure and $p_{\text {calc }}$ is the pressure calculated by the empirical polynomial correlation mentioned above. As seen in Figure 3, the deviation of the data obtained in the present study was within $1.5 \%$. In addition, the absolute average deviation between the data obtained in the present study and the data of Komatsu ${ }^{16}$ was within the same limit. The more considerable difference among the present data and the data of Zhang et al. ${ }^{14}$ and Cruz Duarte et al. ${ }^{15}$ might be caused by the different measuring methods applied in the experiments or the different sizes of the cells and other errors as explained by Komatsu. ${ }^{16}$

The binary hydrate equilibrium data of the systems $\mathrm{CP}+$ water + nitrogen and $\mathrm{CP}+$ water + oxygen were measured experimentally and are reported in Table 2. The measured dissociated conditions and plotted phase boundaries for single nitrogen hydrate and single oxygen hydrate are compared with those for binary nitrogen $+\mathrm{CP}$ hydrate and binary oxygen + $\mathrm{CP}$ hydrate in Figure 4. With the presence of cyclopentane and consequently the formation of the binary hydrate $\mathrm{CP}+$ nitrogen

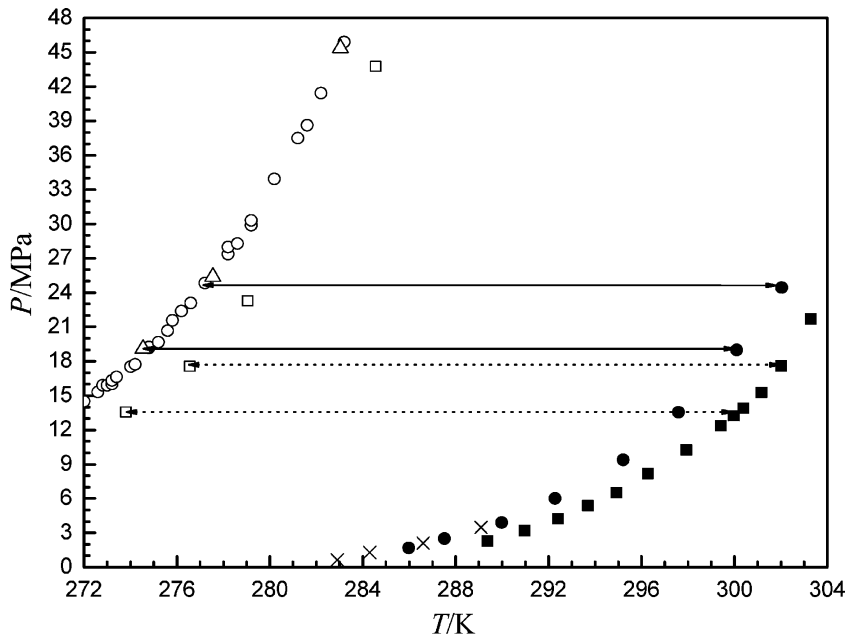

Figure 4. Phase equilibrium data for $(\Delta)$ single nitrogen hydrate, ref 21 ; (O) single nitrogen hydrate, ref 7; ( $\square$ ) single oxygen hydrate, ref 21; (×) binary $\mathrm{CP}+$ nitrogen hydrate, ref $13 ;(\bullet)$ binary $\mathrm{CP}+$ nitrogen hydrate, this work; $(\square)$ binary $\mathrm{CP}+$ oxygen hydrate, this work.

or $\mathrm{CP}+$ oxygen, nitrogen or oxygen can be incorporated into the hydrate cages at lower pressure and higher temperature. As can be seen in Figure 4, the final melting point of the binary nitrogen $+\mathrm{CP}$ hydrate increases by $25.7 \mathrm{~K}$ with respect to that of the single nitrogen hydrate at about $19 \mathrm{MPa}$, whereas the increase of the dissociation temperature reduces to $24.7 \mathrm{~K}$ at 25.2 MPa. The same trend occurs for the $\mathrm{CP}+$ water + oxygen system, as the variation in phase equilibrium temperature of binary oxygen $+\mathrm{CP}$ hydrate relative to single oxygen hydrate reduces from $26.2 \mathrm{~K}$ at $13.5 \mathrm{MPa}$ to $25.5 \mathrm{~K}$ at $17.5 \mathrm{MPa}$. Meanwhile, it can be observed that, at the same pressure of 19.1 MPa, the phase equilibrium curve for binary nitrogen + $\mathrm{CP}$ hydrate shifts by approximately $25.5 \mathrm{~K}$, which is about 0.5 $\mathrm{K}$ higher than that for binary oxygen $+\mathrm{CP}$ hydrate.

\section{Conclusions}

We have reported the four-phase hydrate equilibrium data of the $\mathrm{CP}+$ water + hydrogen, $\mathrm{CP}+$ water + nitrogen, and $\mathrm{CP}+$ water + oxygen systems in the temperature range of 281.3-303.1 $\mathrm{K}$ and the pressure range of 2.27-30.40 $\mathrm{MPa}$. An isochoric pressure-search method ${ }^{18,19}$ was used to perform all of the measurements. The measurements were first performed for the hydrogen $+\mathrm{CP}$ binary clathrate hydrate system, for which there sufficient experimental data are available in the literature. ${ }^{14-16}$ Comparisons between the experimental data measured in this work and the literature data showed acceptable agreement, thus confirming the reliability of the isochoric pressure-search method used in this work. We then reported experimental dissociation data for clathrate hydrates of $\mathrm{CP}$ with nitrogen and oxygen as the helper gases. The dissociation data for the $\mathrm{CP}+$ water + nitrogen system were obtained in extended range of pressure and temperature, and the experimental equilibrium data for the binary hydrate of $\mathrm{CP}+$ water + oxygen are the first ever reported in the literature. As the second guest molecule, $\mathrm{CP}$ showed similar effects for nitrogen and oxygen hydrates in terms of reducing the formation pressure. Moreover, these studies provide valuable information on the practical applications of binary hydrogen, nitrogen, and oxygen hydrates.

\section{Acknowledgment}

This work was supported by the National Natural Science Foundation of China (50876107), National Basic Research 
Program of China (2009CB219504), NSFC-Guangdong Union Foundation (U0733003), and CAS Program (KGCX2-YW-805).

\section{Literature Cited}

(1) Florusse, L. J.; Peters, C. J.; Schoonman, J.; Hester, K. C.; Koh, C. A.; Dec, S. F.; Marsh, K. N.; Sloan, E. D. Stable Low-Pressure Hydrogen Clusters Stored in a Binary Clathrate Hydrate. Science 2004, 306, 469471.

(2) Dyadin, Y. A.; Larionov, E. G.; Manakov, A. Yu.; Zhurko, F. V.; Aladko, E. Ya.; Mikina, T. V.; Komarov, V. Yu. Clathrate Hydrates of Hydrogen and Neon. Mendeleev Commun. 1999, 5, 209-210.

(3) Hashimoto, S.; Sugahara, T.; Sato, H.; Ohgaki, K. Thermodynamic Stability of $\mathrm{H}_{2}+$ Tetrahydrofuran Mixed Gas Hydrate in Nonstoichiometric Aqueous Solutions. J. Chem. Eng. Data 2007, 52, 517-520.

(4) Strobel, T. A.; Koh, C. A.; Sloan, E. D. Water Cavities of sH Clathrate Hydrate Stabilized by Molecular Hydrogen. J. Phys. Chem. B 2008, 112, 1885-1887.

(5) Cruz Duarte, A. R.; Shariati, A.; Peters, C. J. Phase Equilibrium Measurements of Structure sH Hydrogen Clathrate Hydrates with Various Promoters. J. Chem. Eng. Data 2009, 54, 1628-1632.

(6) Fan, S. S.; Liang, D. Q.; Guo, K. H. Hydrate Equilibrium Conditions for Cyclopentane and a Quaternary Cyclopentane-Rich Mixture. J. Chem. Eng. Data 2001, 46, 930-932.

(7) Sloan, E. D.; Koh, C. A. Clathrate Hydrates of Natural Gases, 3rd ed.; CRC Press: Boca Raton, FL, 2008.

(8) Mohammadi, A. H.; Richon, D. Phase equilibria of clathrate hydrates of methyl cyclopentane, methyl cyclohexane, cyclopentane or cyclohexane + carbon dioxide. Chem. Eng. Sci. 2009, 64, 5319-5322.

(9) Imai, S.; Okutani, K.; Ohmura, R.; Mori, Y. H. Phase Equilibrium for Clathrate Hydrates Formed with Difluoromethane + either Cyclopentane or Tetra- $n$-butylammonium Bromide. J. Chem. Eng. Data 2005, 50, $1783-$ 1786.

(10) Ohmura, R.; Takeya, S. Phase Equilibrium for Structure II Hydrates Formed with Krypton Coexisting with Cyclopentane, Cyclopentene, or Tetrahydropyran. J. Chem. Eng. Data 2006, 51, 1880-1883.

(11) Takeya, S.; Yasuda, K.; Ohmura, R. Phase Equilibrium for Structure II Hydrates Formed with Methylfluoride Coexisting with Cyclopentane, Fluorocyclopentane, Cyclopentene, or Tetrahydropyran. J. Chem. Eng. Data 2008, 53, 531-534.
(12) Sun, Z. G.; Fan, S. S.; Guo, K. H.; Shi, L.; Guo, Y. K.; Wang, R. Z. Gas Hydrate Phase Equilibrium Data of Cyclohexane and Cyclopentane. J. Chem. Eng. Data 2002, 47, 313-315.

(13) Danseh, A.; Tohidi, B.; Danesh, A.; Todd, A. C.; Burgass, R. W.; Østergaard, K. K. Equilibrium data and thermodynamic modelling of cyclopentane and neopentane hydrates. Equilibrium data and thermodynamic modelling of cyclopentane and neopentane hydrates. Fluid Phase Equilib. 1997, 138, 241-250.

(14) Zhang, J. S.; Lee, J. W. Equilibrium of Hydrogen + Cyclopentane and Carbon Dioxide + Cyclopentane Binary Hydrates. J. Chem. Eng. Data 2009, 54, 659-661.

(15) Cruz Duarte, A. R.; Shariati, A.; Zevenbergen, J. F.; Florusse, L. J.; Peters, C. J. Hydrogen Storage in Clathrate Hydrates: Phase Equilibria, Thermodynamics and Kinetics. Presented at the 17th World Hydrogen Energy Conference (WHEC2008), Queensland, Australia, Jun 15-19, 2008.

(16) Komatsu, H.; Yoshioka, H.; Ota, M.; Sato, Y.; Watanabe, M.; Smith, R. L.; Peters, C. J. Phase Equilibrium Measurements of HydrogenTetrahydrofuran and Hydrogen-Cyclopentane Binary Clathrate Hydrate Systems. J. Chem. Eng. Data 2010, 55, 2214-2218.

(17) Mohammadi, A. H.; Richon, D. Phase Equilibria of Clathrate Hydrates of Cyclopentane + Hydrogen Sulfide and Cyclopentane + Methane. Ind. Eng. Chem. Res. 2009, 48, 9045-9048.

(18) Liang, D. Q.; Du, J. W.; Li, D. L. Hydrate Equilibrium Data for Methane + tert-Butylamine + Water. J. Chem. Eng. Data 2010, 55, 10391041.

(19) Mohammadi, A. H.; Richon, D. Equilibrium Data of Methyl Cyclohexane + Hydrogen Sulfide and Methyl Cyclohexane + Methane Clathrate Hydrates. J. Chem. Eng. Data 2010, 55, 566-569.

(20) Tohidi, B.; Burgass, R. W.; Danesh, A.; Østergaard, K. K.; Todd, A. C. Improving the Accuracy of Gas Hydrate Dissociation Point Measurements. Ann. N.Y. Acad. Sci. 2000, 912, 924-931.

(21) Mohammadi, A. H.; Tohidi, B.; Burgass, R. W. Equilibrium Data and Thermodynamic Modeling of Nitrogen, Oxygen, and Air Clathrate Hydrates. J. Chem. Eng. Data 2003, 48, 612-616.

Received for review June 23, 2010 Revised manuscript received August 22, 2010 Accepted September 2, 2010

IE101339J 